\title{
SEMISISTEMAS EN ESPACIOS DE HILBERT
}

por

F. castillo

$y$

E. LLORENS

La teorla de sistemas dinamicos discretos ha resultado fitil para el estudio axiomático, asi como para demostrar la convergencia en algunos casos, de los algoritmos de optimización de funciones e incluso de funcionales si se pide a estos condicio nes fuertes de regilaridad.

En el presente trabajo se define un semisistema dinamico dis creto sin unicidad sobre un espacio de Hilbert en el que se suponen ambas topologias, la fuerte deducida de la norma y la débil. Con ello se aborda el estudio de algoritmos de optimización de funcionales cuando sus extremos se alcanzan en puntos que son limite débil de la sucesión definida por el algoritmo. 


\section{I.: PAEL IAINARES}

Sea H un especio real de Hitbert, separeble, cuyo producto interno denotaramos por $(. /$ ). La norma y distancia induci das se simbolizarán respectivamente con $\|$ li $y \quad d(., \ldots)$.

La topologla débil de $\mathrm{H}_{i}, \sigma(\mathrm{HH})$, será designeda por ${ }^{\circ}$, y las conceptos a elle ralativos, se distinguirán con Ios prsfijos ' $\sigma$ ' - 0 "debil" indistintamente.

En lo que sigue $F^{\prime}(H)$ es el conjunto de las partes no vacias $\sigma$-compactes de $H$.

En $F(H)$ se define, dados $A, B \in F(H)$ :

$$
\beta(A, B)=\sup _{x \in A}\{d(x, B)\}
$$

\section{1.- Teorema}

$B(A, B)=0 \Leftrightarrow A \subset B$

$\dot{p}(A, B)<\bar{c} \Longleftrightarrow A C \exists(e, E)$

En general $B(A, B) \neq B(a, A)$

$B(A, B) \leqslant B(A, C)+B(C, B)$

para cualesciuiera $A, B, L \in F(H)$.

La demastración es trivial.

\section{2.- Teqrema}

jea $A \varepsilon F(H)$. $j(A, r)=\{B \in F(H): B(Q A)<r\}$

si ge defing $X 3=\left\{S_{B}(A, r) ; A_{E} F(H) \quad r>0\right\}$

dicha fanilif constituye una base de una tonglogia sgbre $F(H)$, que satisface el orimer axiomil de numerabilided, gra la cual di cho espacio tonológico es no $T_{I}$.

La comprobación es inmediata. 


\section{3.- Tearema}

Ses $\left\{A_{n}\right\} \subset F(H)$ ial que $A_{n} \rightarrow B . A$ A $\varepsilon F(A)$.

Sea $y_{n} \varepsilon A_{3}$ pare $n=1,2, \ldots$

Existe entances una suisucesion $\left\{y_{n_{p}}\right\}$ de $\left\{y_{n}\right\}$ que $\sigma$ - converge a un punto y $\varepsilon A$.

La demostración es'enteraniente anśloga a la del teoreme 0.4 de $(3)$.

\section{Q.4.-Proposicion}

Sean $\left\{x_{n}\right\} \quad\left\{y_{n}\right\}$ sucesiones en $H$ tales que

$$
x_{n} \stackrel{0}{\longrightarrow} x \quad y_{n} \stackrel{a}{\longrightarrow} y
$$

verificando gara cada $n=1,2, \ldots .\left\|x_{n}-y_{n}\right\| \leqslant \varepsilon$

Entonces se tiane sue $\|$ ll $y-x \hbar \leqslant \varepsilon$.

Prucba:

Cono consecuencia de la desigualdad de Cauchy-Schwartz s8 verifica para cada entero positivo 1:

$\|\left(y_{1} / a\right)-\left(x_{1} / a\right)=\left|\left(y_{1}-x_{1} / a\right)\right|\left\{y_{1}-x_{i} \mid U l a \|\right.$ celkal $\quad \forall a \in H$.

Por tanto,

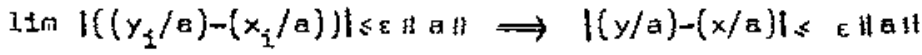

lo cual se curnie sara ceda vector a $\mathrm{E} H$. Tomando

$$
a=\frac{y-x}{\|y-x\|}
$$

quedara:

$$
|(y-x / a)|=\left|\left(y-x / \frac{y-x}{\|y-x\| !}\right)\right|=|| y-x \leqslant \varepsilon\|\theta\|=\varepsilon \quad \text {. }
$$

\section{$2.5 \quad$ Proposicion}

Gean $8 \in F(H), \quad S[B, \epsilon]=\{x \in H: d(x, \exists)<\varepsilon\}$

intoncess $S[3, \varepsilon] E F(: 1\}$.

\section{Prucbe}

jee $\quad\left[x_{n}\right\} \subset s[E, \varepsilon]$.

com $5\left[f_{f}\right]$ es acotado, al ser $H$ reflexivo; ss debilmente relativenente secuencialmente comacto, jor lo aue la sucesion dado admite uno suisucosión $\left\{x_{n_{p}}\right\}$ lébilmente convemente 
a un ounto XE H.

Como para cada $p, x_{n_{p}} s[B, \epsilon]$ se tiene que $d\left(x_{n_{p}}, \theta\right)=a_{n_{p}} \leqslant \varepsilon \quad$.

Al ser $B$ dabilmente campacto y el conjurto $\left\{x_{n_{p}}\right\}$ cerrado convexo ( Jara cada o), pusob afirmerse, como consecuencia del enunciedo 26.2.日. de (z) que existe $y_{n_{p}} \varepsilon$ e, tal que of e. $\left.x_{n_{p}}\right)=\left[y_{n_{p}}-x_{n_{p}}=\alpha n_{p} \& \varepsilon\right.$.

i.B sucesion $\left\{y_{n_{p}}\right\}$ esta contenida en $B$, debilmente compacto; fur lo que admite una subsucesion, (puade suponerse ella misma) que converge déblimente a un punto $y \in B$. Como $x_{n_{p}} \stackrel{a}{\rightarrow} x$ se verifican las hiptesis de le propistcion antertor, por to que $\|y-x\| \leqslant \varepsilon$

$y$ de aqul que $x \in \mathrm{s}[\mathrm{\theta}, \varepsilon]$ probéndose asi que $\mathrm{s}[\mathrm{\theta}, \varepsilon]$ es débilmente secuenctalmente compacto.

AI ser $H$, obviamente un espacio de frechet, se sigue la conclusión del teorema como consecuencla de (2) 24,3,9. 


\section{1.- DEFINICIOH}

Se denomine o-semisistema dinámico discrets sin unicidad sobre $H, a$ la terna $\left(H, I^{+}\right.$, T. $)$, donde

$$
\begin{aligned}
& \pi: H x I^{+} \longrightarrow F(H) \quad \text { verifica: } \\
& 2.1 .1 .-\quad \pi(x, 0)=\{x\} \\
& 2.1 .2 .-\quad \pi(\pi(x, k), h)=\pi(x, k+h) \quad \forall k, h \varepsilon I^{+} \forall x_{\varepsilon} H \\
& 2.1 .3 .-\quad \pi: H \longrightarrow F(H), \pi k_{k}(x)=\pi(x, k) \text {, es }
\end{aligned}
$$

$\sigma-\beta$ - contínua para cada $k$ entero no negativo.

En lo que sigue se supane dedo un a -semisistema dinâ fiico discreto $\left(H, I^{+}, \pi\right)$ sobre $H$.

\section{2.- Definicion}

La aplicación $x: J \longrightarrow H$ es una solución del

- -semidistema dinámico discreta, a través, de $x_{\varepsilon} H$ si verifica:

$$
\begin{aligned}
& 2.2 .1 .-I^{+} \subset J \\
& 2.2 .2 .-x(0)=x \\
& 2.2 .3 .-x(j)=\pi(x(k), j-k) \quad \forall j, k \in I^{+} \quad j>k .
\end{aligned}
$$

\section{3.- Teoreme}

Jea $y \varepsilon \pi(x, k) . k \in I^{+}$. Entances existe en $I^{+}$una so-

lución $x$ por $x$ tal tue $x(k)=y$.

Es su prueba idéntica a la del Teorema 1.1 .1 de (3).

2.4. - Jefinicion

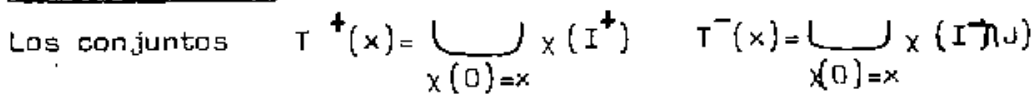

son llamados semiconos de trayectorias, positivo y nogativo res nectivanente, a través de $\times$ EH.

$\because$ cono de trayectorias a través de $x$, rerá

$T(x)=T^{+}(x)$ u $T^{-}(x)$.

\section{5. - Definición}

ji $\{x\}=T(x)$, x es llamerlo unto crítico. 
Un conjunto $M \subset M$ se dice:

2.6.1.- Positivenente invariante, si $T^{*}(k)=$ i.j.

2.6.2.- Negativamente invariante, si $T^{\&}(\mathrm{H}-\mathrm{H})$ - H-M.

2:6.3.- Débilmente positiverente invariante si para todo gunto $y \in: a$, existe una solución $x$ a través de $y$, tal rue $x\left(I^{\&}\right) \subset M$.

2.6.4.- Débilmente negativemente Invariante si nara todo punto $y \in$ i.i,existe una solución $x$, tal qye $x\left(\mathrm{~J} \cap \mathrm{I}^{-}\right) \subset \mathrm{l}$.

\section{7.- Teorema}

Condicion necesaria y suficiente para que el conjunto $\mathrm{ACH}$ sea dobilimente gosftivamente invariente es que para todo $x \in$ i:i,

$$
\pi(x, 1) \cap k \neq \phi
$$

\section{Priteda}

jea $x_{0} E$ i., arbitraria. $\pi\left(x_{g}, 1\right) \cap n \neq \phi$, morlo : ve existe $x_{1} \in \pi\left(x_{0}, 1\right)$, con $x_{1}$ en :.i, de donde $\pi\left(x_{1}, 1\right) \cap::$ t. oor lo rue existe $x_{2}$ en $4, x_{2} \in \pi\left(x_{2}, 2\right)$. Reiterando el razonamiento canstrui mos una sucesion $\left\{x_{i}\right\}$ con $x_{i} \in$ ग $\left(x_{i-1}, 1\right)$, contenida en : .

$$
x: I^{+} \longrightarrow H, x(i)=x_{i}
$$

es, abviaiente, la soluck'tin buscrida. 1 resta de le prueba es trivinl

\section{8.: Definteion}

Se llame conjunto norma-1imita positiva de la trayectaria recorrido de la solución $x$, al

$L^{\phi}(x)=\left\{y \varepsilon H\right.$ : existe una sucesión $\left\{k_{n}<I_{n}^{+} k_{n} \underset{n}{x}\left(k_{n}\right)+y\right\}$ 2.3.- Definición

Se llame conjunto o-l1mite oositivo de la trayectorio recorrido de la solución $x$, al

$L_{\sigma}^{+}(x)=\left(y \in H\right.$ : exista una sucesión $\left.\left\{k_{n}\right) \subset I^{b}, k_{n}+\infty \times\left(k_{n}\right) \stackrel{\sigma}{\rightarrow} y\right\}$

\subsection{0.- Observación}

Se tendra que $L^{\&}(x)<L_{0}^{+}(x)$.

En edelante se enplasr. indistintamente el simbolo ' $x$ ', oare hacer reforencia a una sọución $\supset$ a su corresjondiente reco rrido $\supset$ trayectoria. 
je consirira la ajlicacisn $x^{+}: H \longrightarrow 2^{H}$ dofinida como stgue:

$$
\lambda^{+}(x)=\left\{\begin{array}{l}
\$ \text { si existe } u n_{i} ; \text { soluci in } x \text { zov } x, \text { tal aue } I^{+}(x)=\phi \\
\underbrace{}_{x(0)=x} L^{+}(x) \text { en casn contrario }
\end{array}\right.
$$

Pera cade elemento $x \in H, \lambda^{t}(x)$ se denomina conjunto dorma-lsmite no sitivo de $x$.

\section{a..12.- Oefinición.}

Se considera la aplicacion $\lambda_{0}^{+}: H \longrightarrow z^{H}$ definida como sigue:

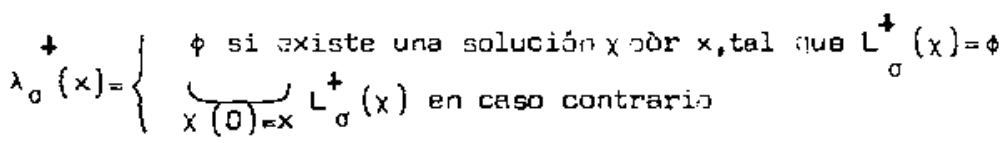

Fara cada elemento $x$ de $H, \lambda_{0}{ }^{\dagger}(x)$ se demomine conjunto o-limite Dositivi de $\times$,

\subsection{3.- Observación}

je tiene que $\lambda^{t}(x) \subset \lambda_{\sigma}^{t}(x)$ sace cada $x \in H$.

\subsection{3.- Tenrema}

$\mathrm{L}^{+}(x)$ es norma-cerrada y débilanente positivanente invariante.

Pruede

je encuencra en (4) Th.3.3.

\subsection{Teorema}

Si $x$ es solución por $x \in H$ tal que $T^{+}(x)$ E $F\left(H^{+}\right)$, entonces $L_{\sigma}^{+}(x)$ \& $F(H)$.

\section{Prueba}

$L_{f}^{*}(x) \notin \phi$ parque $\{x(i)\}$ es una sucesión en $T^{+}(x)$, que admitirá alguna subsucesión convergente. Sea $\left\{y_{n}\right\}$ una sucesion en $L_{0}^{*}(x), y_{n} \stackrel{\sigma}{\rightarrow} y$. Existen sucesiones de enteros no neçativos, $\left\{k_{p}^{n}\right\}$ tales que $x\left(k_{p}^{n}\right) \rightarrow y_{n} \quad n=1,2, \ldots$. Ccmo $T^{4}(x)$ es acotado, su tofalogia relativa a la debil de $H$ puecie definirse por una métrica $d^{*}(, \ldots)$. Podemos entances suporer que las sucesiones $\quad\left(x\left(k_{p}^{n}\right)\right\}$ verifican: $\left.d^{*}\left(x\left(k_{p}^{n}\right), y_{n}\right)\right) \leqslant \frac{1}{p}$ y $k_{n}^{n}<k_{n-1}^{n-1}$. Es facil ver ahore que $x\left(k_{n}^{r}\right) \rightarrow y$, por lo que $L_{\sigma}^{+}(x)$ es débilmente secuencialmente cerrado. La conclusión se sigue ahora de (2),24.1.7. 
Sea $V \subset H$.

Sea $\$: V \longrightarrow A$

\$ es una función de Lianunov ara el a -semisistethe dinámico discreta dado, si y tílo si

2.15.1.- \$ es débilmente secuéncialmente contínua.

2.15.2.- $\Phi(x) \geqslant \Phi(x(1))$ pata torla solución $x$ tal Qu日 $x(1) \in V, \quad x(0)=x \in V$.

\subsection{6.- Teorema}

Sea $\$$ una función de Liadunov para el a-semisistema dinămico discreto dado, definida en un conjunto $V \subset H$, débil mente secuencialmente cerrado.

Entonces, si $x$ es cualquier salución de trayectoria contenida en $V$, y tal sue $L_{\phi}^{+}(x) \neq \phi$, se verifica que $\phi$ está definida $y$ es constante solzre $L_{\sigma}^{+}(x)$.

\section{Pruebs}

Gear $y_{1} z$ elementos de $L_{0}^{+}(x)$. tales gue $\phi(y)<\phi(z)$.

Pueden encontrarse das sucesiones $\{k\}\left\{h_{n}\right\}$, con

$k_{n} \rightarrow+\infty \quad h_{n}+\infty$ tales cive $x\left(k_{n}\right) \stackrel{\sigma}{\rightarrow} z \quad x\left(n_{n}\right) \stackrel{0}{\longrightarrow} y$.

inbas sucesiones meden ser tamadas de nodo que

$n_{n}<k_{n} n=1,2, \ldots$, oor lo que para cada $n$ se tiene

$$
\phi\left(x\left(k_{n}\right)\right) \leqslant \Phi\left(x\left(h_{n}\right)\right\}
$$

de donde $\lim \varphi\left(x\left(k_{n}\right)\right) \leqslant \lim \Phi\left(x\left(h_{n}\right)\right)$. De 2.15 .1 se oitione que $\phi(z) \leqslant \phi(y)$, lo cual es contracictorio.

il estar la trayectoria de $x$ contenta en $V y$ éste ser débimente secuencialnente cerrado, se tencirá que si $\times \varepsilon L_{\sigma}^{+}(x)$ es un punto de acumulación détsil de $V, y$ gar lo tanto, $x \in V$, lo ique completa $1 \%$ demostracion. 
$L_{f}^{+}(x)$ es débilmente positiva aente invariante, perit cada solución $x$.

\section{Prueba}

Seg $y \in L_{g}^{+}(x)$.

Por definición existe una sucesión $\left\{k_{n}\right\} \subset I^{+}, k_{n}^{++\infty}$, tal que $x\left(k_{n}\right) \stackrel{g}{\rightarrow} y$.

$$
\begin{aligned}
& \text { Entonces, } x\left(k_{n}+1\right) \varepsilon \pi\left(x\left(k_{n}\right), 1\right) \\
& \text { Song } \pi(x)=\pi(x, 1) \text { es } 0-\beta-\text {-continua, } y \quad x\left(k_{n}\right) 9 \text { y, se }
\end{aligned}
$$

tendré que $\pi\left(x\left(k_{n}\right), 1\right) \stackrel{B}{\rightarrow} \pi_{[}(y, 1), y$ como consecuencia dal teorema 1.3: existe una subsucesion $\left\{k_{n}^{*}+1\right\}$ de $\left\{k_{n}+1\right\}$ tal nue

$$
\begin{aligned}
& x\left(k_{n}^{*}+1\right) \rightarrow z \varepsilon \pi(y, 1) . \\
& \text { Como } k_{n}^{*}+1 \rightarrow+\infty, z \varepsilon L_{\sigma}^{+}(x) . \\
& \text { Luego, para toda } y \in L_{\sigma}^{+}(x) \text {, se cumple que } \pi(y, 1) \cap L_{\sigma}^{+}(x) \neq \Phi
\end{aligned}
$$

lo que en virtud de 2.7 completa la demostración.

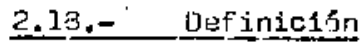

Sea ine $F(H)$.

2.13.1.- Se dice que ti es norma-3stable si dado cualquier norma-antorno $U$ de $M$, existe un norna entorno $V$ de $M$, positivamente invariante, contenido en $U$.

2.18.2.- Se dice que es $\sigma_{\text {mestable si dado cualquier }}$ a-entorno $\mathrm{U}$ de $:$, existe un $\sigma$-entorno $V$ de $i$, positivanente invatian te, contentzdo en $U$.

\subsection{9.- Definición}

उen $M \in F(i-1)$.

2.19.1.- Se dice que $\$$ es norma-atractor d6bil cuanda existe un norme-entorno $U$ de $M$, tal que para todo $\times E U$, se verifica:
i) $\lambda_{\sigma}(x) \neq \phi$
ii) $x(x) \subset i$

2.19.2.- Se dice ruc if es a -atractor débil cuando existe un o-entarno $U$ de $k$, tal que para toda $x \in U$ se verifica:
i) $\lambda_{\sigma}(x) \neq \phi$
ii) $\lambda(x)<t .1$ 
Sag $M E F(H)$.

2.20.1. - Se dice que $M$ as nome asintbticaments estable

si es norma-estable y norma-atractor dóbil.

2.20.2.- Se dice que M es o-asintoticanente estable 51

es $\sigma$-estable y $\sigma$-atractor débll.

2.20.3.- Se dice que M es glogalmente asintoticanente

estable (en norma), si es norma-asintótioamente estable y $\forall x \in H$ se verifica

i) $\lambda_{\sigma}(x) \neq \phi \quad$ ii) $\lambda_{\sigma}(x) \subset M$

2.20.4.- Se dice que $M$ as o-globelmente asłnt6t1camen-

te estable si es o-astable, $y \quad \forall \times c h$ se verifica:
1) $\lambda_{\sigma}(x) \neq \phi$
ii) $\lambda_{\sigma}(x)<M$. 
Sean the $F(H)$, Y $V \in H$.

Si $\$: V \rightarrow R$ es una función de Liapunov pare

el б-s8misistema dinámico dado, vertficando:

1) $V$ es positivamente invariants.

2) $\Phi(x)=0 \quad \forall x \in A . \quad \Phi(x)>0 \quad \forall x \in \vee-M$.

3) उi $x$ E-ki,nara tada solucion $x$ por $x, \phi(x(I))<\phi(x)$.

4) Para todo norna-entorno $U$, de $A, U C V$, existe $B$ \&q,

$\beta>0$, tai nus $\phi(x) \geqslant \beta \times \varepsilon v-J$,

entonces $H$ es norina-asintóticamente estable.

Prueba

a) id es norma-estable

Sea $U$ un norma-entorno de $M$. Iomamos $\alpha>0$, tal que

$\mathrm{S}[\mathrm{M}, \alpha] \mathrm{CU}, \mathrm{S}[\mathrm{H}, \alpha] \quad F(H)$ nor 1.5 .

Sea $m=\inf \{4(x)\}$. Por $(4), m>0$. $x \in V-S[-1, a]$

Tomando $K=\{x \in S[M p]: \Phi(x) \leq m / z ;$ se ve que es debil mente compacto, entorno de $i, y$ positivamente inveriants, pues si $x \in k y$ $x$ es una solucion por $x$, il ser $V$ aositivante invarian te esté definido $\phi(x(2))$, y por (3) se tiene que $\Phi(x(1))<\Phi(x) \in m / 2$ de donde $x(1) \varepsilon k$.

b) Mes norma-etractor débil

jea $x \in k$, Si $x$ es Eualquier solucion con $x(0)=x$, entonces

$\{x(i\})$ es una sucesión de elenentos de $k$ y al ser este debilmente compacto adfite una subsucesión debilmente convergente a un punto yek,or lo que para dicha solución $L_{\alpha}^{f}(x) \neq \$$, y por tanto

$\forall x \varepsilon k, \lambda{ }_{\sigma}^{t}(x) \neq \phi$.

ii $y \in \lambda^{+}(x)-l i$, existe una solución $x$ nor $x$, tal que ye $L_{0}^{+}(x)-M$. Lano $\mathfrak{L}_{0}^{+}(x)$ es débilmente positivanente invariante, exis te una solución $\zeta$, por $y$, tal ciue $\zeta(1) \varepsilon \vec{L}_{0}^{+}(x)$. Unt inces por $(j)$, se cumplecque $\phi(\zeta(1))<\phi(y)$, lo cual es absurdo oues $\phi$ es constante sobre $L_{\sigma}^{+}(x)$.

Por tanto $x_{d}^{+}(x)<\mu, \forall x k$, lo Liuo ormingta la orueba. 
S1 $H \in F(H), y \quad V \subset H$, see $\$: V \rightarrow \rightarrow A$ una funcion de Liapunóv para el $\sigma$-semidistema dinàmico discrato dado, verificando:

$$
\begin{aligned}
& \text { 2.22.1.: } \quad V \text { es norme entorno de } M . \\
& \text { 2.22.2.: } \quad V \text { es positivamante invariante. } \\
& \text { 2.22.3.: } \quad V E F(H) \\
& \text { 2.22.4.: Para coda solucion } x \text { por } x \in V-M, \\
& \phi(x(1))<\quad(x) . \\
& \text { Entonces } \quad \text { es norme atractor dóbil. }
\end{aligned}
$$

Demostrac1on:

S1 $x \in V$ y $x$ es una solucion por $x$, entances $\{x(i)\}_{\text {if }} I^{*}$ es una sucesion en $V, y$ podemos guponer, por 2.22 .3 que

$$
x(i) \stackrel{\sigma}{\rightarrow} \text { y. } E \text {. }
$$

Por lo tanto $L_{\sigma}^{+}(x) \neq \phi, y$ camo $x$ es arbitrarie $\lambda^{+}(x) \neq \phi$.

Los restantes datalles son identicas a los de la demostración del teorema anterior, y par tanto se omitan.

$$
2.23 .: \text { COROLARIO }
$$

Con Iguales hiptesis y notaciones que en el teorsma ante rior, supongamos que $\$$ tiens un único minimo en $x^{\pi} \varepsilon V$. Entonces, si $\left\{x^{a}\right\}=M$ verifica 2.22 .4 , as norma atractor debil. 


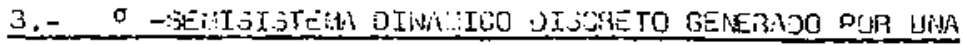

APLICACION UE H EN $F(i) \quad \sigma-B$ EOTITINA

Sea $f(., 1): H \rightarrow F(H)$ una eplicación $\sigma-8 \rightarrow$ contfrua.

Definimos $f: H x I^{+}: \longrightarrow f(H) \quad\left\{\begin{array}{l}f(x, 0)=\{x\} \\ f(x, k)=\underbrace{}_{y \in f(x, k-1)} f(y, I)\end{array}\right.$

\section{1.- Teorena}

f está bien defirida.

rueba

-Basta probar que si K es un conjunto débilmante compacto contenido en $H, i d=\underbrace{}_{y \in K} f(y, 1)$, también lo es.

sea $\left\{x_{n}\right\} C h$. Por construccion rueble abtenerse otra sucesion corresiondiente, $\left(y_{n}\right) \subset K$, tal que $x_{n} \varepsilon f\left(y_{n}, 1\right)$.

il ser $k$ debilnimte secuenciblmente compacto, existe en $K$ una subsucesión de $y_{n},\left\{y_{n}^{*}\right\}$ cébilmente convergente a un punto yei.

Por ser $f \sigma-\beta-$ continua, $f\left(y_{n}^{*}, 1\right) \rightarrow-\beta \rightarrow f(y, 1), y$ cano $x_{n}^{*} \varepsilon f\left(y_{n}^{*}, 1\right)$; nor el tcoreial 1.3 :xiste una suisucesion $\left\{x_{n}^{*}\right\}$ que norma-converge, $y$ jor canto es debilizente convergente, a un punto $x \varepsilon f(y, 1)$; como $y \in K, \quad x \varepsilon \bigcup_{y \in K} f(y, 1)$, con lo cual se prueba que is :s débilinente secuencialmente comacto, y de aqui (Cfr. (2) 24.3.:.), que es débilnente compacto.

3.2.- Teorema

$f(x, k+h)=f(f(x, k), h), \forall x \pi, \forall h, k \varepsilon I^{+}$

Pruebe

Es identhca a la del teorema II.2.2. de (3).

3.3.- Teorema

$f_{k}: H \longrightarrow F(H), f_{k}(x)=f(k, k)$ bs $a-\beta$-secuenciilikente continua para cada $k \varepsilon I^{+}$.

Prueba

ie hará jor inilucción sobre !.

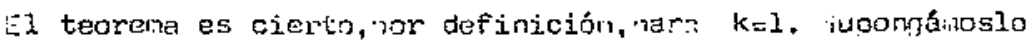
cierto para k-1. 
Sea $\left\{x_{n}\right\}$ uns sucesion obbilmentc convervente a $x \in H$.

Surongamos que en tal caso $f\left(x_{n}, k\right) \rightarrow f \rightarrow f(x, k)$.

Ello quiera dacir que existe $0>0, y$ una sucesion $\left\{x_{n}\right\}$ de forme que $8\left(f\left(x_{n_{i}}, k\right), f(x, k)\right) \geqslant \rho$. Tomendo $z_{n_{j}} \in f\left(x_{n_{i}}, k\right)$ de modo que $d\left(z_{n_{i}}, f(x, k)\right) \geqslant 0$, 7uede obtenerse una sucesión $\left(y_{n_{i}}\right\}$ con $y_{n_{i}} \in f\left(x_{n_{i}}, k-1\right)$, de suerte que $z_{n_{1}} \in f\left(y_{n_{i}}, 1\right)$.

Como $x_{n_{j}} \stackrel{\sigma}{\rightarrow} x$, por hipótesis de inducción deber cumplirse aue $f\left(x_{n_{j}}, k-t\right)-B \rightarrow f(x, k-1)$, y por el teorema 1.3 existe una subsucesion $\left\{y_{n_{i}^{*}}^{*}\right\}$ de $\left\{y_{n_{i}}\right\}$ tal que norma-converge a un sunto $y \in f(x, k-1)$, de donde

$$
f\left(y_{n_{ \pm}}^{*}, 1\right) \stackrel{\beta}{\rightarrow} f(y, 1)
$$

y como $z_{n_{i}} \varepsilon f\left(y_{n_{i}}, 1\right)$ aplicando nuevamente 81 teoreme 1.3 se tiene que existe $z \varepsilon f(y, I)$ que es el norma-limite de una subsucesión $\left\{z_{n_{i}}^{e+}\right\}$ def $z_{n_{1}}^{*}$.

Como yef(x,k-1), $f(y, 1) \subset f(f(x, k-1), 1)=f(x, k)$, lwego $z \in f(x, k)$, con io que $d\left(z, z_{n_{i}}\right) \geqslant \rho$ la que contradice al hecho de gue $\left\{z_{n_{i}}^{\prime \prime}\right\}$ norna-converge $a z$.

\section{4.- Corglarto}

En las condiciones del teoreina anterior, $f_{k}$ es continua para cada $K \varepsilon I^{4}$.

\section{5.- Corolario}

$\left(H, I^{+}, f\right)$ constituye un o.-semisistema dinámico discreto sogre H. 
Sea $B=\left\{v_{4}, v_{2}, v_{3}, \ldots, v_{f}\right\} \quad$, un conjunto finito de vectores de $H, B \neq\{0\}$.

$$
\begin{aligned}
& \text { Oefinimos } f(., I): H \longrightarrow F(H) \text {, de modo que } \\
& f(x, I)=\left\{\left(x / v_{i}\right) v_{i}, i=1, \ldots, f\right\}
\end{aligned}
$$

See $x_{n} \varepsilon$ t una sucesión débilmente convergente a $\times E H$. Por definicion sc tiene entonces que dado $\varepsilon>0$, existe $n(\xi) \in I^{+}$, tal que pare todo vector $w \in H, \quad\left|\left(x_{n} / w\right)-(x / n)\right|<\varepsilon / \max \left\|\mid v_{1}\right\|^{\natural}, n \geqslant n(\xi)$. Entances, para todo $n \geqslant n(\varepsilon)$,

$$
\beta\left(f\left(x_{n}, I\right), f(x, I)\right)=\max _{y \in f\left(x_{n}, 1\right)}\{d(y, f(x, I)\})=
$$

$\left.=\max _{y \in f\left(x_{n}, I\right)}\left\{\min _{1}\left\{\| y-\left(x / v_{i}\right) v_{i} \mid\right\}\right\} \leqslant \max _{y \in f\left(x_{n}, 1\right)}\left\{H y-\left(x / v_{i}\right) v_{i} \|\right\} \quad(i=1 \ldots j\}\right\}$ $=\left\|\left(x_{n} / v_{p}\right) v_{n}-\left(x / v_{p}\right) v_{p}\right\|=\left\|\left(x_{n}-x / v_{p}\right)\right\|\left\|v_{p}\right\|<\frac{\varepsilon}{\max \left\|v_{f}\right\|}\left\|v_{p}\right\| \leqslant \varepsilon$ Luggo, $f(., 1)$ es $\sigma-\beta-\operatorname{cont} 1$ nue $y$ como consecuencle, $\left(H, I^{+}, f\right)$ un $\sigma$-senisistema dinámica discreto sobre $H$.

En particular, si $B$ es ortonormal, $y k \in I^{+}, k>0$, $\{0\} \cup f(x, I)=f(x, k)$, si $j>1$.

it $B$ fuera ortogonal y se tuviera ademas que $l i v_{1} \|=\lambda_{ \pm}<1$, es fácil ver que, para $j>1, f(x, k)=\{0\} \quad u\left\{\lambda_{i}^{2(k-1)}\left(x / v_{1}\right) v_{i}, 1 \ldots 1 \ldots j\right\}$ por lo uue st $x_{i}$ es una solución por $x$ tal que $x_{i}(k)=\lambda_{1}^{2(k-1)}(x / v i) v_{i}$ $(k>0)$, antancas $L^{t}\left(x_{1}\right)=\{0\}$.

\section{7.- Ejemglo}

Sea $H=3_{n}^{2}, y\left\{\theta_{n}\right\}$ su base artonornal canonica.

Definimos $f(\ldots, 1): H \rightarrow f(H), f(x, I)=3[0,1]$.

Esta alicación es, trivialmente $\sigma-\beta$-contánua, jor lo que $\left(H, I^{+}, f\right)$ ss un G-semisistena dinámico discreto soture $H$.

ji $x \in h$, guede definirse $x(0)=x, x(k)=e_{k} k \varepsilon I^{+}$.

$X$ es una solución del satisisteme que verifica :

$$
L^{+}(x)=\$, L_{\sigma}^{+}(x)=\{0\} \text {. }
$$


(1) DeI Gastillo, f.: "Senisistemas dinúnicos discretos" Publ1c. Fac. C1encias 1.Compl. adrid. i-171.

(2) Kathe, G.: "Topological Vector Spaces $\mathrm{I}^{*}$ Sprimer-varleg, 1.969 .

(3) Hodrlguez i.:- "Aplicaciones de la Teorfa de Jenisjstenas Dinánicas Oiscretos a ciertos Algoritmos de iinimización" Public.0to.Anglisis Hat. U.Santisgo de Lompostels

(4) Szego-Treccent.: "An abstract formudation of minimization elgorithms" 